\title{
Approach and Means of Support for Mental Disorders Caused by the Traumatic Amputation of a Limb
}

\author{
Bogdan Alexandru Ciubară, Ovidiu Alexa, Lucian Ștefan Burlea, \\ Ilinca Untu, Anamaria Ciubară \\ University of Medicine and Pharmacy Gr. T. Popa, Iași, Romania \\ Email: ilinca tzutzu@yahoo.com
}

Received January 2015

\begin{abstract}
Amputation, one of the oldest types of surgery performed for therapeutic purposes, has main indication, the severe trauma to a limb that is incompatible to preserve because of the irreversible ischemia. The loss of a limb due to trauma has also a devastating emotional impact on a patient, escalating to genuine mental disorders that stretch out further than the loss of the limb itself produces. Thus, there is a certain sequential order regarding risk for developing mental disorders, starting from the immediate reaction, the acute stress disorder, and ending with the post-traumatic stress disorder, depression and anxiety that is mostly related to one's perceived image, which further aggravates their disability. The present paper aims to realize a synthesis of the emotional elements that can intensify up to mental disorders; by understanding the impact of squeal produced by the amputation, we can actually improve the multidisciplinary approach before and after surgery for the patient that suffered an amputation.
\end{abstract}

\section{Keywords}

Amputated Limb, Post-Traumatic Stress Disorder, Acute Stress Disorder, Depression, Anxiety

\section{Introduction}

The loss of a limb in a traumatic context is indubitably an unexpected, unpredictable and emotionally devastating event. Most frequently, these patients were in good health when the trauma occurred, fully functional and productive on all levels [1]. Therefore, the traumatic loss of a limb determines a radical transformation these individuals' existence, with a deep impact on their global functionality [2]. The loss of a limb causes, beyond the physical disability, an important emotional disability, which may degenerate to the development of psychiatric pathologies [1].

\section{Generalities on the Nature of Amputation}

The traumatic amputation of a limb is the underlying cause of acute stress disorder, as well as of posttraumatic

How to cite this paper: Ciubară, B.A., Alexa, O., Burlea, L.Ș., Untu, I. and Ciubară, A. (2015) Approach and Means of Support for Mental Disorders Caused by the Traumatic Amputation of a Limb. Open Journal of Social Sciences, 3, 21-24.

http://dx.doi.org/10.4236/jss.2015.36005 
stress disorder, depression and anxiety [1].

The amputation of a limb represents the irreversible loss of an extremity, (regardless of its level) consecutive to a trauma or a surgery required by various medical conditions. Common situations leading to limb amputations are malign or benign bone or soft tissue tumours, severe and unmonitored limb infections, peripheral vascular disease and, of course, traumatic lesions. The only absolute indication for limb amputation is irreversible ischemia on an affected extremity or on an extremity that suffered severe trauma. Whereas, in case of organic, tumoral, and infectious underlying factors, amputation is planned, in case of traumatisms, it is unplanned. Among young patients, traumatisms are the main cause of amputations [2].

\section{Specific of Psychiatric Disorders in Patients with Traumatic Limb Amputation}

The loss of a limb due to trauma has also a devastating emotional impact on a patient, escalating to genuine mental disorders that stretch out further than the loss of the limb itself produces. Thus, there is a certain sequential order regarding risk for developing mental disorders, starting from the immediate reaction-the acute stress disorder - and ending with the post-traumatic stress disorder, depression and anxiety that is mostly related to one’s perceived image, which further aggravates the disability [1]-[3].

\subsection{Acute Stress Disorder}

During the acute period, in the first month following the trauma that led to limb amputation, patients are prone to develop acute stress disorder, which lasts at least two days, but it can go on for four weeks [1]. Acute traumatic stress disorder is determined by the subjective experience of the individual concerning a profoundly traumatizing event, which leads to an extreme state of stress that inhibits the coping mechanisms of the individual [4]. Approximately three quarters of the individuals who develop acute stress disorder are likely to develop posttraumatic stress disorders; the individuals who are more prone to it are those who associated dissociative elements to the disorder [3].

\subsection{Posttraumatic Stress Disorder}

Posttraumatic stress disorder is a psychiatric disorder caused by exposed to a life-threatening situation; a defining characteristic of the traumatizing event is that the person's response to it supposes intense fear and a felling of helplessness or horror [3]. Considering the intensity of feelings associated with the trauma, its perception can be distorted; there may be various degrees of dissociation between affects and trauma and even partial or total amnesia concerning the psycho-traumatizing event per se. The mark of posttraumatic stress disorder is the subjective reprise of initial symptoms and their intrusive character. The re-experiencing of the traumatic event is vivid, accompanied by the initial flow of bodily sensations, which the patient is unable to control. Troubled sleep, full of terrifying, repetitive nightmares of a quasi-real character is frequent symptoms. The individual can also have flashbacks that lead him to think he is reliving the traumatic event all over again. The entire flow of psychiatric symptoms is accompanied by the hyperactivity of vegetative system [1] [3] [5]. The physical disability provoked by the unexpected loss of a limb is worsened by a state of detachment from reality, from the significant others, by one's personal pre-morbid motivational systems. Posttraumatic stress disorder may have an acute evolution (less than three months) or it may become chronic (exceeding three months); in the second case, it may leave behind residual symptoms or it may be reactive, years apart from a seeming remission [3]. Irrespective of its manifestations forms, this psychiatric disorder affects the global functionality of the individual who suffered a limb amputation, thus delaying or even stopping (sometimes irreversibly) the individual from sociofamilial and professional reintegration or reinsertion (depending on what the individual did for a living before the traumatic event) [1] [5].

Research demonstrates that planned surgeries for limb amputation-for therapeutic purposes, determined by various medical conditions with chronic evolution, which causes local ischemia—only rarely lead to posttraumatic stress disorder. On the contrary, unpredictable, traumatic events that involve the unplanned amputation of a limb lead to increased rates of posttraumatic stress disorder, fundamentally due to the shock provoked by the trauma itself [6].

\subsection{Anxiety and Depression}

Furthermore, persons who went through limb amputation because of a traumatic event are very prone to a hy- 
perthymic negative symptomatology; depression is a frequent pathology among such individuals. In this context, depression sets off as a maladaptive response to the surgery and especially to the sudden and striking loss that led to a real disability [1] [6]. The most stringent aspect, which triggers depressive symptomatology, is precisely the incapacity of reintegrating in pre-morbid activities [1]. Therefore, persons who made a living within mostly physical activities before the trauma adapt harder and it takes them longer to deal with the loss of a limb, compared to individuals who were sedentary before the trauma and who could resume their professional lives without great difficulties [7] [8].

The interval of time since the amputation correlates significantly and inversely proportionally with the anxiety perceived by the patients. At the same time, age at amputation correlates in an inversely proportional manner with the level of adaptability to sudden loss; the younger the patients, the more difficult to cope after being condemned to disability [1].

\subsection{Factors That Influence the Coping Mechanisms}

Coping mechanism for the sudden loss of a limb consecutive to a trauma are profoundly influenced by the potential local pain, by the level of disability, the cosmetic aspects, the cultural clichés, the presence of social and familial support systems, etc. It is also worth mentioning the patient's pre-morbid capacity of deal with problems. Most frequently, the cosmetic aspect of the remnant is a decisive factor in the development and maintaining of psychiatric symptoms, besides the dysfunctionality per se [5] [9].

Thus far, studies have failed to determine precisely whether posttraumatic stress disorder is the direct consequence of the traumatic incident, of the sudden loss of the limb, or of the global context provided by the two events together [1].

\section{Discussions and Conclusions}

By understanding the impact of sequelae produced by the amputation, we can actually improve the multidisciplinary approach before and after surgery for the patient that suffered an amputation, thus helping them adjust better to their new limiting status.

Individuals who suffered traumatic limb amputations experience severe and long-term difficulties on physical and mental level, which have a great impact upon their general health status, especially upon their quality of life; in this sense, perceived self-image is a fundamental factor.

This paper aims at warning with regard to the mental risks involved in the traumatic amputation of a limb, which often leads to patients' inability of resuming their social and professional life; in addition, it is a genuine threat to their quality of life. Studies have proved the increase in the incidence of immediately posttraumatic acute stress disorder and of posttraumatic stress disorder, of reactive depression and of anxiety, mostly related to perceived body image. The issue is all the more serious as it concerns the young, active population members, who suddenly have to cope with an unexpected situation, which brings along a radical change that turns their entire existence and their system of values upside down. It is worth underscoring the need to monitor the mental status of patients with posttraumatic amputations, in order to discover early and treat all psychopathological incidents. We believe that, in the future, specific multidisciplinary support systems should be elaborated for the patients integrated within this population, taking into account the risks entailed by omitting the psychiatric side that, by its nature, can determine an aggravation of the individual's disability. It can also delay their rehabilitation and, in dramatic cases, it may lead to suicide, when reactive depression has an increased severity.

\section{References}

[1] Copuroglu, C., Ozcan, M. and Ymlaz, B. (2010) Acute Stress Disorder and Post-Traumatic Stress Disorder Following Traumatic Amputation. Acta Orthopaedica Belgica, 76, 90-93.

[2] Heck, R.K. (2008) General Principles of Amputations. In: Canale, S.T. and Beaty, J.H., Eds., Campbell's Operative Orthopaedics, 11th Edition, Mosby Elsevier, Philadelphia, 561-578. http://dx.doi.org/10.1016/B978-0-323-03329-9.50012-X

[3] American Psychiatric Association (2013) DSM-5. Diagnostic and Statistical Manual of Mental Disorders.

[4] Cavanagh, S.R., Shin, L.M., Karamouz, N. and Rauch, S.L. (2006) Psychiatric and Emotional Sequelae of Surgical Amputation. Psychosomatics, 47, 459-464. http://dx.doi.org/10.1176/appi.psy.47.6.459

[5] Desmond, D.M. and MacLachlan, M. (2006) Coping Strategies as Predictors of Psychosocial Adaptation in a Sample 
of Elderly Veterans with Acquired Lower Limb Amputations. Social Science Medicine, 62, 208-216. http://dx.doi.org/10.1016/j.socscimed.2005.05.011

[6] Ebrahimzadeh, M.H. and Rajabi, M.T. (2007) Long Term Outcomes of Patients Undergoing War-Related Amputations of the Foot and Ankle. Journal of Foot and Ankle Surgery, 46, 429-433. http://dx.doi.org/10.1053/j.jfas.2007.08.011

[7] Bhuvaneswar, C.G., Epstein, L.A. and Stern, T.A. (2007) Reactions to Amputation: Recognition and Treatment. Journal of Clinical Psychiatry, 9, 303-308. http://dx.doi.org/10.4088/PCC.v09n0408

[8] Ferguson, A.D., Richie, B.S. and Gomez, M.J. (2008) Psychologicalfactors after Traumatic Amputation in Landmine Survivors: The Bridge between Physical Healing and Full Recovery. Disability and Rehabilitation, 14-15, 931-938.

[9] Sumer, A., Onur, E. and Altınlı, E. (2008) Our Clinical Experiences after Lower Extremity Amputations. Inönü Üniversitesi Tip Fakültesi Dergisi, 15, 187-190. 